\title{
PROJECTIVE MODULAR REPRESENTATIONS OF FINITE GROUPS II
}

\author{
by J. F. HUMPHREYS
}

(Received 19 September, 1979)

In this paper, which is a continuation of [4], the necessary theoretical background is given to enable the calculation of the irreducible Brauer projective characters of a given finite group to be carried out. As an example, this calculation is done for the alternating group $A(7)$ in $\$ 3$. In a future paper the calculations for the Mathieu groups will be presented.

Let $K$ be any field and $K^{\times}$denote its multiplicative group. Given a finite group $G$, a mapping $\alpha: G \times G \rightarrow K^{\times}$is a $K$-cocycle if

$$
\alpha(x, y) \alpha(x y, z)=\alpha(x, y z) \alpha(y, z)
$$

and

$$
\alpha(x, 1)=1=\alpha(1, x)
$$

for all $x, y, z \in G$. An $\alpha$-projective representation of $G$ of degree $n$ over $K$ is a map $P: G \rightarrow G L(n, K)$ such that for all $x, y \in G$

$$
P(x) P(y)=\alpha(x, y) P(x y)
$$

and

$$
P(1)=I,
$$

where $I$ denotes the identity $n \times n$ matrix.

The twisted group algebra $K(G, \alpha)$ is a $K$ vector space with basis $\{(g) \mid g \in G\}$ and multiplication obtained by extending by linearity that on the basis elements given by $(x)(y)=\alpha(x, y)(x y)$. Thus an $\alpha$-projective representation of $G$ is the representation afforded by some $K(G, \alpha)$-module. Under suitable restrictions on $K$, there is a group $H$, which is a central extension of $G$, such that, for suitable $\alpha$, an $\alpha$-projective representation of $G$ can be linearized by a representation of $H$. This fact is used, in Section 1 , to investigate the relationship between the principal indecomposable submodules of $K(G, \alpha)$ and those of $K(H)$. In Section 2, the results of Section 1 are used to establish the orthogonality relations for Brauer projective characters.

1. Principal indecomposable projective representations. Let $G$ be a finite group and $K_{0}$ be the algebraic number field obtained by adjoining a primitive $|G|$ th root of unity to the rationals. Let $R_{0}$ be the algebraic integers of $K_{0}$ and $\mathscr{P}_{0}$ be the ideal of $R_{0}$ containing the rational prime $p$. Denote by $\nu_{p}$ the $p$-adic valuation associated with $\mathscr{P}_{0}$, normalized so that $\nu_{\mathrm{p}}(p)=1$. Let $R$ denote the completion of $R_{0}$ with respect to $\nu_{\mathrm{p}}, \mathscr{P}$ denote the corresponding prime ideal and $K$ be the field of quotients of $R$. Write $\bar{K}$ for the field $R / \mathscr{P}$, so that $\bar{K} \cong R_{0} / \mathscr{P}_{0}$ and let the coset of any element $x$ of $R$ modulo $\mathscr{P}$ be $\bar{x}$.

Glasgow Math. J. 22 (1981) 89-99. 
Now let $\tilde{H}$ be a complex representation group of $G$ so that $\bar{H}$ has a central subgroup $\tilde{A}$ with $\tilde{A} \leq \tilde{H}^{\prime}$ such that $\tilde{A} \cong H^{2}\left(G, \mathbb{C}^{\times}\right)$and there is an isomorphism $\tilde{\sigma}: G \rightarrow \tilde{H} / \tilde{A}$. For each $g \in G$, choose an element $\tilde{r}(g)$ of $\tilde{H}$ such that $\tilde{\sigma}(g)=\tilde{r}(g) \tilde{A}$, with the convention that $\tilde{r}(1)$ is the identity element of $\tilde{H}$. Thus, if $x, y \in G$, since $\tilde{\sigma}$ is a homomorphism,

$$
\tilde{r}(x) \tilde{r}(y)=\tilde{r}(x y) \tilde{A}(x, y)
$$

for some $\tilde{A}(x, y) \in \tilde{A}$. Hence, if $\lambda \in \operatorname{Hom}\left(\tilde{A}, \mathbb{C}^{\times}\right)$, the map $\alpha: G \times G \rightarrow \mathbb{C}^{\times}$defined by

$$
\alpha(x, y)=\lambda(\tilde{A}(x, y))
$$

for all $x, y \in G$, is a complex 2-cocycle which we call the special cocycle associated with $\lambda$. Since the values of $\lambda$ are in $R_{0}$, and therefore in $R$, the map $\bar{\alpha}$ defined by

$$
\bar{\alpha}(x, y)=\overline{\alpha(x, y)}
$$

for all $x, y \in G$, is a $\bar{K}$ 2-cocycle of $G$.

Next, let $k$ be the algebraic closure of $\bar{K}$ and $\tilde{A}_{p}$ be a Sylow $p$-subgroup of $\tilde{A}$. It is proved in [1], that $\boldsymbol{H}=\tilde{H} / \tilde{A}_{p}$ is a $k$-representation group of $G$. Thus, denoting $\tilde{A} / \tilde{A}_{\mathrm{p}}$ by $A$ and the coset $\tilde{h} \tilde{A}_{\mathrm{p}}$ by $h$, we have that $A \cong H^{2}\left(G, k^{\times}\right)$and the map $\sigma: G \rightarrow H / A$ defined by $\sigma(g)=r(g) A$, for all $g \in G$, is an isomorphism. Also, writing $\tilde{A}(x, y) \tilde{A}_{p}$ as $A(x, y)$, we have that

$$
r(x) r(y)=r(x y) A(x, y) .
$$

For $\lambda \in \operatorname{Hom}\left(\tilde{A}, \mathbb{C}^{\times}\right)$, we may define $\bar{\lambda} \in \operatorname{Hom}\left(A, \bar{K}^{\times}\right)$by the rule

$$
\bar{\lambda}(a)=\overline{\lambda(\tilde{a})}
$$

for all $a \in A$. Thus the cocycle $\bar{\alpha}$ defined above is the special cocycle associated with $\bar{\lambda}$ in the sense that, for all $x, y \in G$,

$$
\bar{\alpha}(x, y)=\bar{\lambda}(A(x, y)) .
$$

For any $R$-free $R$-algebra $B$, let $\bar{B}$ be the $\bar{K}$-algebra $B / B \mathscr{P}$. Notice that if $B$ is $R(G, \alpha)$, the twisted group algebra of $G$ over $R$ with cocycle $\alpha$, then $\bar{B}$ is $\bar{K}(G, \bar{\alpha})$. Let $W_{1}, \ldots, W_{r}$ be a complete set of representatives for the isomorphism classes of principal indecomposable $R(G, \alpha)$-modules. By $[3 ; 44.3], V_{1}, \ldots, V_{r}$ is a complete set of representatives for the isomorphism classes of principal indecomposable $\bar{K}(G, \bar{\alpha})$-modules, where $V_{i}=\bar{W}_{i}(1 \leq i \leq r)$. Let $L_{i}=V_{i} / \operatorname{rad}\left(V_{i}\right)(1 \leq i \leq r)$, so that, by $[3 ; 45.8], L_{1}, \ldots, L_{r}$ is a complete set of representatives for the isomorphism classes of irreducible $\bar{K}(G, \bar{\alpha})$ modules. Since $R(G, \alpha) \otimes_{R} K$ may be identified with $K(G, \alpha),[3 ; 48.1]$ implies that there exists a set $P_{1}, \ldots, P_{s}$ of finitely generated $R$-free $R(G, \alpha)$-modules such that every irreducible $K(G, \alpha)$-module is isomorphic to exactly one $P_{i} \otimes_{R} K$.

Defintrions. (i) Let $d_{i j}$ be the number of times $L_{j}$ occurs as a composition factor of $\bar{P}_{i}$. The matrix $D=\left(d_{i j}\right)$ is the decomposition matrix of $R(G, \alpha)$.

(ii) Let $c_{i j}$ be the number of times $L_{j}$ occurs as a composition factor of $\bar{W}_{i}=V_{i}$. The matrix $C=\left(c_{i j}\right)$ is the Cartan matrix of $\bar{K}(G, \bar{\alpha})$. 
The following is immediate from $[3 ; 48.8]$.

THEOREM 1.1. With the above notation

(i) $W_{i} \otimes_{R} K \cong \bigoplus_{i=1}^{r} d_{j i}\left(P_{j} \otimes_{R} K\right)$; and

(ii) $C=D^{\mathrm{T}} D$, so that $C$ is a symmetric matrix.

Let $\bar{\alpha}$ be the special cocycle associated with $\bar{\lambda}$. The twisted group algebra $\bar{K}(G, \bar{\alpha})$ may be regarded as a $(\bar{K}(H), \bar{K}(G, \bar{\alpha}))$-bimodule with obvious right action and, for $a \in A$, $g \in G, v \in \bar{K}(G, \bar{\alpha})$,

$$
\operatorname{ar}(g) v=\bar{\lambda}(a)(g) v
$$

Thus, if $V$ is any $\bar{K}(G, \bar{\alpha})$-module,

is a $\bar{K}(H)$-module.

$$
V_{H}=\bar{K}(G, \bar{\alpha}) \underset{\bar{K}(G, \bar{\alpha})}{\otimes V}
$$

Proposition 1.2. Let $\bar{\alpha}$ be the special cocycle associated with $\bar{\lambda}$. Let $V$ be a principal indecomposable $\bar{K}(G, \bar{\alpha})$-module. Then $V_{H}$ is isomorphic to a principal indecomposable $\bar{K}(H)$-module $U$ and for each $a \in A$, a acts on $U$ as multiplication by $\bar{\lambda}(a)$. Conversely, if $U$ is a principal indecomposable $\bar{K}(H)$-module such that for $a \in A$, a acts on $U$ as multiplication by $\bar{\lambda}(a)$, then there exists a principal indecomposable $\bar{K}(G, \bar{\alpha})$-module $V$ such that $V_{H}$ is isomorphic to $U$.

Proof. Let $e$ be the idempotent of $\bar{K}(A)$ corresponding to $\bar{\lambda}$, so that for $a \in A$, $a e=\bar{\lambda}(a) e$, and let

$$
\bar{K}(H, \bar{\lambda})=\bigoplus_{g \in G} \bar{K} r(g) e
$$

It is clear that $\bar{K}(H, \bar{\lambda})$ is a $\bar{K}$-subspace of $\bar{K}(H)$. However, it is also a $\bar{K}(H)$-submodule, since for $a \in A, x, y \in G$,

$$
\begin{aligned}
\operatorname{ar}(x)(r(y) e) & =a A(x, y) r(x y) e \\
& =r(x y) a A(x, y) e \\
& =r(x y) \bar{\lambda}(a) \bar{\lambda}(A(x, y)) e \\
& =\bar{\lambda}(a) \bar{\alpha}(x, y) r(x y) e .
\end{aligned}
$$

Let $\theta: \bar{K}(G, \bar{\alpha})_{H} \rightarrow \bar{K}(H, \bar{\lambda})$ be the $\bar{K}$-linear map defined on basis elements by

$$
\theta(1 \otimes(g))=r(g) e .
$$


Then $\theta$ is a $\bar{K}(H)$-module isomorphism, since for $a \in A, x, y \in G$

$$
\begin{aligned}
\theta(\operatorname{ar}(x)(1 \otimes(y))) & =\theta(\bar{\lambda}(a)((x) \otimes(y))) \\
& =\bar{\lambda}(a) \theta(1 \otimes(x)(y)) \\
& =\bar{\lambda}(a) \theta(1 \otimes \alpha(x, y)(x y)) \\
& =\bar{\lambda}(a) \alpha(x, y) r(x y) e \\
& =a A(x, y) r(x y) e \\
& =\operatorname{ar}(x) r(y) e \\
& =\operatorname{ar}(x) \theta(1 \otimes(y)) .
\end{aligned}
$$

It is clear from the above that for each $a \in A, a$ acts on $\bar{K}(H, \bar{\lambda})$ as multiplication by $\bar{\lambda}(a)$. Now if $V$ is an indecomposable summand of $\bar{K}(G, \bar{\alpha}), V_{H}$ is an indecomposable summand of $\bar{K}(G, \bar{\alpha})_{H}$. Thus $\theta\left(V_{H}\right)$ is an indecomposable summand of $\bar{K}(H, \bar{\lambda})$. Since $\bar{K}(H)$ has a $\bar{K}(H)$-module decomposition

$$
\bar{K}(H)=\oplus \bar{K}(H, \bar{\mu})
$$

as $\bar{\mu}$ ranges over $\operatorname{Hom}\left(A, \bar{K}^{\times}\right)$, we see that $\theta\left(V_{H}\right)$ is a principal indecomposable of $\bar{K}(H)$.

Conversely, if $U$ is a principal indecomposable of $\bar{K}(H)$, equation (1) shows that $U$ is a summand of $\bar{K}(H, \bar{\mu})$ for some $\bar{\mu} \in \operatorname{Hom}\left(A, \bar{K}^{\times}\right)$. In this case, for $a \in A, a$ acts on $U$ as multiplication by $\bar{\mu}(a)$. Therefore, if for each $a \in A, a$ acts on $U$ as multiplication by $\bar{\lambda}(a)$, $U$ is a summand of $\bar{K}(H, \bar{\lambda})$. Thus $\theta^{-1}(U)$ is a principal indecomposable $\bar{K}(G, \bar{\alpha})_{H^{-}}$ module. Hence there is a principal indecomposable $K(G, \bar{\alpha})$-module $V$, say, that $V_{H}$ is isomorphic to $U$.

COROLLARY 1.3. Let $L_{1}, \ldots, L_{r}$ be a complete set of representatives for the isomorphism classes of $\bar{K}(G, \bar{\alpha})$-modules. Then $\left(L_{1}\right)_{H}, \ldots,\left(L_{r}\right)_{H}$ is a complete set of representatives for the isomorphism classes of those irreducible $\bar{K}(H)$-modules on which each $a \in A$ acts as multiplication by $\bar{\lambda}(a)$.

Proof. It is easily shown that $\bar{K}(G, \bar{\alpha})$ is a Frobenius algebra and so each principal indecomposable $\bar{K}(G, \bar{\alpha})$-module has a unique minimal submodule which is irreducible. the result now follows by Proposition 1.2.

THEOREM 1.4. With a suitable ordering of the modules for $H$, the Cartan matrix of $\bar{K}(G, \bar{\alpha})$ is a direct submatrix of the Cartan matrix of $\bar{K}(H)$.

Proof. Let $U_{1}, \ldots, U_{n}$ be the principal indecomposable $\bar{K}(H)$-modules with the ordering chosen so that $U_{i}=\theta\left(\left(V_{i}\right)_{H}\right)$ for $1 \leq i \leq r$, where $\theta$ is the map used in the proof of Proposition 1.2, and $V_{1}, \ldots, V_{r}$ are the principal indecomposable $\bar{K}(G, \bar{\alpha})$-modules. Let $L_{i}$ be the unique minimal submodule of $V_{i}(1 \leq i \leq r)$ and $M_{i}$ be the unique minimal submodule of $U_{j}(1 \leq j \leq n)$. Because of the way $A$ acts on $M_{i}$ and $U_{j}$, it follows that for $1 \leq i \leq r$ the only composition factors of $U_{i}$ are those in the set $M_{1}, \ldots, M_{r}$. Furthermore, if $L_{j}$ occurs $k$ times as a composition factor of $V_{i}$ then $M_{j}$ occurs $k$ times as a composition factor of $U_{i}$. This proves the result. 
Corollary 1.5. Let $C$ be the Cartan matrix of $\bar{K}(G, \bar{\alpha})$. Then $\operatorname{det} C$ is a power of p.

Proof. By $[3 ; 61.5]$, the determinant of the Cartan matrix of $\bar{K}(H)$ is a power of $p$. The result follows by Theorem 1.4 .

To conclude this section, we obtain a similar result to Theorem 1.4 for the decomposition matrix of $R(G, \alpha)$. Let $\alpha$ be the special cocycle associated with $\lambda$. Then $R(G, \alpha)$ may be regarded as an $(R(\tilde{H}), R(G, \alpha))$-bimodule with natural right action and, for $\tilde{a} \in \tilde{A}$, $g \in G, v \in R(G, \alpha)$,

$$
\tilde{a} \tilde{r}(\mathrm{~g}) v=\lambda(\tilde{a})(g) v
$$

Thus if $M$ is any $R(G, \alpha)$-module

is a $K(\tilde{H})$-module.

$$
M_{\bar{H}}=R(G, \alpha) \otimes_{R(G, \alpha)} M
$$

Proposition 1.6. Let $P_{1}, \ldots, P_{s}$ be a set of finitely generated $R$-free $R(G, \alpha)$-modules such that every irreducible $K(G, \alpha)$-module is isomorphic to exactly one $P_{i} \otimes_{R} K$. For $1 \leq i \leq s$, let

$$
\hat{P}_{i}=\left(P_{i}\right)_{\dot{H}} \otimes_{R} K
$$

Then $\hat{P}_{1}, \ldots, \hat{P}_{s}$ is a complete set of representatives for the isomorphism classes of those irreducible $K(\tilde{H})$-modules on which elements $\tilde{a}$ of $\tilde{A}$ act as multiplication by $\lambda(\tilde{a})$.

Proof. It is clear that each $\hat{P}_{i}$ is an irreducible $K(\tilde{H})$-module on which each element $\tilde{a}$ of $\tilde{A}$ acts as multiplication by $\lambda(\tilde{a})$. There is a one-to-one correspondence between irreducible $\alpha$-projective representations of $G$ over $K$ and those irreducible representations of $K(\tilde{H})$ which restrict to $A$ as $\lambda$ times the identity matrix. This gives a one-to-one correspondence between irreducible $K(G, \alpha)$-modules and those irreducible $K(\tilde{H})$ modules on which elements $\tilde{a}$ of $\tilde{A}$ act as multiplication by $\lambda(\tilde{a})$. Thus, in order to show that for $i \neq j, \hat{P}_{i}$ is not isomorphic to $\hat{P}_{j}$, it is sufficient to prove that if $D$ is an irreducible $K(\tilde{H})$-module on which each element $\tilde{a} \in \tilde{A}$ acts as multiplication by $\lambda(\tilde{a})$ then $D$ is isomorphic to $\hat{P}_{i}$ for some $i$. By $[3 ; 48.1]$, there is an $R$-free $R(\tilde{H})$-module $Q$ such that $D=Q \bigotimes_{R} K$. Now $Q$ may be regarded as $Q_{1}$, an $R(G, \alpha)$-module, by defining for $g \in G$, $v \in Q$

$$
(g) v=r(g) v .
$$

Then $Q_{1}$ is $R$-free and $\left(Q_{1}\right)_{\tilde{H}}$ is isomorphic to $Q$. Also $Q_{1} \otimes K$ is an irreducible $K(G, \alpha)$-module and so is isomorphic to $P_{i} \otimes_{R} K$ for some $i$. Thus

$$
D=Q \bigotimes_{R} K \cong\left(Q_{1}\right)_{\bar{H}} \otimes_{R} K \cong\left(P_{i}\right)_{\bar{H}} \otimes_{R} K=\hat{P}_{i},
$$

proving the result.

In a similar way to the proof of Theorem 1.4 , one can now prove

THEOREM 1.7. With a suitable ordering of the modules for $\tilde{H}$, the decomposition matrix of $R(G, \alpha)$ is a direct submatrix of the decomposition matrix of $K(\tilde{H})$. 
2. Character relations. The Brauer projective characters $\psi_{1}, \ldots, \psi_{r}$ of $L_{1}, \ldots, L_{r}$ are defined as follows. Let $\phi_{1}, \ldots, \phi_{r}$ be the Brauer characters of the irreducible $\bar{K}(H)$-modules $\left(L_{1}\right)_{H}, \ldots,\left(L_{r}\right)_{H}$ and define, for $g \in G$

$$
\psi_{i}(g)=\phi_{i}(r(g))
$$

Similarly, if $\eta_{1}, \ldots, \eta_{r}$ are the Brauer characters of $\left(V_{1}\right)_{H}, \ldots,\left(V_{r}\right)_{H}$, the Brauer projective characters $\mu_{1}, \ldots, \mu_{r}$ of $V_{1}, \ldots, V_{r}$ are defined by

$$
\mu_{i}(g)=\eta_{i}(r(g))
$$

It follows by Proposition 1.2 and Theorem 4.1 of [4] that if

$$
\eta_{i}=\sum_{j=1}^{r} c_{i j} \phi_{j}
$$

then

$$
\mu_{i}=\sum_{j=1}^{r} c_{i j} \psi_{j}
$$

An element $g$ of $G$ is $\bar{\alpha}$-regular if $\bar{\alpha}(g, x)=\bar{\alpha}(x, g)$ for all $x \in C_{G}(\mathrm{~g})$. If an element of $G$ is $\bar{\alpha}$-regular so are all its conjugates. A conjugacy class consisting of $\bar{\alpha}$-regular elements is called an $\bar{\alpha}$-regular conjugacy class. By Theorem 4.2 of [4], the number of pairwise non-isomorphic irreducible $\bar{K}(G, \bar{\alpha})$-modules is equal to the number of $\bar{\alpha}$-regular $p$ regular conjugacy classes of $G$. Let $x_{1}, \ldots, x_{r}$ be representatives for these classes.

Given complex valued functions $f, g$ on $G$ let

$$
\langle f, g\rangle^{\prime}=\frac{1}{|G|} \sum_{x \in G_{0}} f(x) \overline{g(x)},
$$

where $G_{0}$ denotes the set of $p$-regular elements of $G$. Finally, writing $C=\left(c_{i j}\right)$ for the Cartan matrix of $\bar{K}(G, \bar{\alpha})$, we can state the projective modular orthogonality relations.

THEOREM 2.1. With the above notation,

(i) $\left\langle\mu_{i}, \psi_{j}\right\rangle^{\prime}=\delta_{i j}$;

(ii) $\left\langle\mu_{i}, \mu_{i}\right\rangle^{\prime}=\left\langle\mu_{i}, \mu_{i}\right\rangle^{\prime}=c_{i j}$;

(iii) $\left\langle\psi_{i}, \psi_{j}\right\rangle^{\prime}=b_{i j}$, where $\left(b_{i j}\right)=C^{-1}$; and

(iv) $\sum_{i=1}^{r} \mu_{i}\left(x_{k}\right) \overline{\psi_{i}\left(x_{j}\right)}=\left|C_{G}\left(x_{j}\right)\right| \delta_{i k}$.

Proof. Since the map $\sigma: G \rightarrow H / A$ defined by $\sigma(g)=r(g) A$ is an isomorphism, and $A$ is a $p^{\prime}$-group, if $g$ is $p$-regular then $r(g)$ is $p$-regular as is $\operatorname{ar}(g)$ for any $a \in A$. Conversely if $\operatorname{ar}(g)$ is a $p$-regular element of $H$ then $g$ is a $p$-regular element of $G$. Now let $f, g$ be complex valued functions on the p-regular elements $G_{0}$ of $G$ and $h, k$ be complex valued functions on the p-regular elements $H_{0}$ of $H$ such that for all $a \in A, x \in G$

$$
\begin{aligned}
& h(\operatorname{ar}(x))=\lambda(a) f(x), \text { and } \\
& k(\operatorname{ar}(x))=\lambda(a) g(x),
\end{aligned}
$$


where $\lambda \in \operatorname{Hom}\left(A, \mathbb{C}^{\times}\right)$. Then

$$
\begin{aligned}
\frac{1}{|H|} \sum_{y \in H_{0}} h(y) \overline{k(y)} & =\frac{1}{|H|} \sum_{\substack{a \in A \\
x \in G_{0}}} h(\operatorname{ar}(x)) \overline{k(a r(x))} \\
& =\frac{1}{|H|} \sum_{\substack{a \in A \\
x \in G_{0}}} \lambda(a) \overline{\lambda(a)} f(x) \overline{g(x)} \\
& =\frac{1}{|H|} \sum_{a \in A} \lambda(a) \overline{\lambda(a)} \sum_{x \in G_{0}} f(x) \overline{g(x)} \\
& =\frac{|A|}{|H|} \sum_{x \in G_{0}} f(x) \overline{g(x)} \\
& =\langle f, g\rangle^{\prime} .
\end{aligned}
$$

Thus, (i), (ii) and (iii) follow from the results in $\$ 1$ together with the corresponding, well-known, results in $H$.

In order to prove (iv), it should first be noted that without loss of generality, it may be supposed that Brauer projective characters are class functions. This may be ensured by making a suitable choice for the transversal $\{r(g) \mid g \in G\}$. Once this is done, (iv) follows from (i) and Theorem 4.2 of [4] using a similar proof to the one for the corresponding result for linear characters.

Corollary 2.2. $\nu_{P}(|G|)=\min _{1 \leq i \leq r}\left(\mu_{i}(1)\right)$.

Proof. By 1.2, $\mu_{i}$ has the same degree as a principal indecomposable Brauer character $\eta_{i}$ of $H$. Since $A$ is a $p^{\prime}$-group, $l=\nu_{\mathrm{p}}(|H|)=\nu_{\mathrm{p}}(|G|)$ and so, by [3; 59.7], $p^{l}$ divides $\eta_{i}(1)$. However, evaluating 2.1(iv) at $x_{k}=1=x_{j}$, we see that no higher power of $p$ can divide each $\mu_{i}(1)$.

Now let $P_{1}, P_{2}$ be projective representations of $G$. For $g \in G$ define $\left(P_{1} \otimes P_{2}\right)(\mathrm{g})$ to be the Kronecker product of $P_{1}(g)$ and $P_{2}(g)$. The following result follows easily from the definitions

Proposition 2.3. Let $P_{i}$ be a projective representation of $G$ with special cocycle $\bar{\alpha}_{i}$ and $\psi_{i}$ be the Brauer projective character of $P_{i}(i=1,2)$. Then $P_{1} \otimes P_{2}$ is a projective representation of $G$ with special cocycle $\bar{\alpha}_{1} \bar{\alpha}_{2}$ and the Brauer projective character $\psi$ of $P_{1} \otimes P_{2}$ is given by

$$
\psi(g)=\psi_{1}(g) \psi_{2}(g)
$$

Proposition 2.4. Let $P$ be a projective representation of $G$ over $\bar{K}$ with special cocycle $\bar{\alpha}$ and $\psi$ be the Brauer projective character of $P$. The complex conjugate $\bar{\psi}$ of $\psi$ is the Brauer projective character of a projective representation $P^{*}$ of $G$ over $\bar{K}$ with special cocycle $\bar{\alpha}^{-1}$.

Proof. Let $D$ be the linear representation of $H$ defined by

$$
D(\operatorname{ar}(g))=\bar{\lambda}(a) P(g)
$$


and let $\phi$ be the Brauer character of $D$ so that

$$
\psi(g)=\phi(r(g))
$$

for all $g \in G$. Then the complex conjugate $\bar{\phi}$ of $\phi$ is the Brauer character of the representation $D^{*}$ of $H$ defined by $D^{*}(h)=D\left(h^{-1}\right)^{\mathrm{T}}$. For $\mathrm{g} \in G$, define $P^{*}(\mathrm{~g})$ to be $D^{*}(r(g))$. Then for $x, y \in G$.

$$
\begin{aligned}
P^{*}(x) P^{*}(y) & =D^{*}(r(x)) D^{*}(r(y)) \\
& =D^{*}(r(x) r(y)) \\
& =D^{*}(A(x, y) r(x y)) \\
& =D\left(A(x, y)^{-1} r(x y)^{-1}\right)^{T} \\
& =\bar{\lambda}\left(\bar{A}(x, y)^{-1}\right) D\left(r(x y)^{-1}\right)^{T} \\
& =\bar{\alpha}^{-1}(x, y) P^{*}(x y) .
\end{aligned}
$$

Clearly, for $g \in G$, the Brauer character $\psi^{*}$ of $P^{*}$ satisfies

$$
\psi^{*}(g)=\phi^{*}(r(g))=\bar{\phi}(r(g))=\overline{\phi(r(g))}=\bar{\psi}(g) .
$$

Now let $\bar{\alpha}$ be a special cocycle and $\mu_{1}^{\bar{\alpha}}, \ldots, \mu_{r}^{\bar{\alpha}}$ be the Brauer characters of the principal indecomposable $\bar{\alpha}$-projective representations and $\psi_{1}^{\bar{\alpha}}, \ldots, \psi_{r}^{\bar{\alpha}}$ be the Brauer characters of the irreducible $\bar{\alpha}$-projective representations.

THEOREM 2.5. Let $\bar{\alpha}, \bar{\beta}, \bar{\gamma}$ be special cocycles with $\bar{\alpha} \bar{\beta}=\bar{\gamma}$. Suppose

$$
\psi_{i}^{\bar{\alpha}} \psi_{j}^{\bar{\beta}}=\sum_{k} a_{i j k} \psi_{k}^{\bar{\gamma}}
$$

then

$$
\mu_{i}^{\bar{\gamma}} \psi_{j}^{\bar{\beta}-1}=\sum_{k} a_{k j i} \mu_{k}^{\bar{\alpha}}
$$

where, by Proposition 2.4, $\psi_{j}^{\bar{\beta}-1}$ is the complex conjugate of $\psi_{j}^{\bar{\beta}}$.

Proof. Linearize all the underlying representations to linear representations of $H$. The corresponding formulae hold in $H$ by a result of Brauer and Nesbitt $[2 ;(64)$ and (76)]. The result now follows in view of the fact that, by Proposition $2.3, \psi_{i}^{\bar{\alpha}} \psi_{i}^{\bar{\beta}}$ is expressible in terms of $\psi_{\mathrm{k}}^{\bar{\gamma}}$ since $\bar{\alpha} \bar{\beta}=\bar{\gamma}$.

3. Example. In this section, the Brauer projective characters of the group $A(7)$ are calculated. Schur [6] has shown that $H^{2}\left(G, \mathbb{C}^{\times}\right)$has order six when $G$ is $A(7)$. Thus, if we consider the representations of $G$ modulo $2, \tilde{H}$ has order $6|A(7)|$ and $H$ has order $3|A(7)|$. The first task is to determine the irreducible Brauer projective characters, which may be regarded as irreducible Brauer projective characters of the group $H=3 . A(7)$. Schur gives the ordinary projective character table for $A(7)$ corresponding to a cocycle $\beta$ 
of order 3 . This table is the following

\begin{tabular}{|r|r|r|r|r|r|r|}
\hline 1 & $(2)^{2}$ & $(2)(4)$ & $(5)$ & $(2)^{2}(3)$ & $(7)$ & $(7)^{-1}$ \\
6 & 2 & 0 & 1 & 2 & -1 & -1 \\
15 & -1 & -1 & 0 & 2 & 1 & 1 \\
15 & 3 & 1 & 0 & 0 & 1 & 1 \\
21 & 1 & -1 & 1 & -2 & 0 & 0 \\
21 & -3 & 1 & 1 & 0 & 0 & 0 \\
24 & 0 & 0 & -1 & 0 & $\lambda_{1}$ & $\lambda_{2}$ \\
24 & 0 & 0 & -1 & 0 & $\lambda_{2}$ & $\lambda_{1}$ \\
\hline
\end{tabular}

where $\lambda_{1}=\frac{1}{2}(-1+i \sqrt{ } 7)$ and $\lambda_{2}=\bar{\lambda}_{1}$. Also the conjugacy classes of cycle type (3) and (3) ${ }^{2}$ are $\beta$-irregular and so all the $\beta$-projective characters are zero on those classes. The Brauer characters of $A(7)$ modulo two are well-known (see [5]) and are as shown below.

\begin{tabular}{|r|r|r|r|r|r|}
\hline 1 & $(3)$ & $(3)^{2}$ & $(5)$ & $(7)$ & $(7)^{-1}$ \\
1 & 1 & 1 & 1 & 1 & 1 \\
4 & -2 & 1 & -1 & $1+\lambda_{1}$ & $1+\lambda_{2}$ \\
4 & -2 & 1 & -1 & $1+\lambda_{2}$ & $1+\lambda_{1}$ \\
6 & 3 & 0 & 1 & -1 & -1 \\
14 & 2 & -1 & -1 & 0 & 0 \\
20 & -4 & -1 & 0 & -1 & -1 \\
\hline
\end{tabular}

By Theorem 4.2 of [4], there are four irreducible Brauer $\beta$-projective characters. The two $\beta$-projective characters of degree 24 may be linearized by characters of $H$ and so, since 8 is the highest power of two dividing $|H|$, their restriction to 2 -regular conjugacy classes gives two irreducible Brauer projective characters. There is a six-dimensional $\beta^{-1}$-projective character, which we denote by $\overline{6}$, whose values, by 2.4 , are the same as those of the six-dimensional $\beta$-projective character 6 . By 2.3 , the product character $6 . \overline{6}$ is a character of $A(7)$. Its restriction to 2-regular conjugacy classes can be expressed as $2.1+14+20$ and it therefore follows that 6 is an irreducible Brauer projective character. It therefore only remains to find one further irreducible Brauer projective character.

Suppose 6 were a constituent of 15 as Brauer characters. Then their difference 9, would be a Brauer character taking the value -2 on elements of order 7 . The product of 9 with either of the four-dimensional Brauer characters of $A(7)$ would be a Brauer $\beta$-projective character of degree 36 taking the value $-1-i \sqrt{ } 7$ on a conjugacy class of elements of order 7. This is impossible so 15 is irreducible and the irreducible Brauer 
$\beta$-projective characters of $A(7)$ are
1
(5) (7) $\quad(7)^{-1}$

\begin{tabular}{|r|r|r|r|}
\hline 6 & 1 & -1 & -1 \\
15 & 0 & 1 & 1 \\
24 & -1 & $\lambda_{1}$ & $\lambda_{2}$ \\
24 & -1 & $\lambda_{2}$ & $\lambda_{1}$ \\
\hline
\end{tabular}

The ordinary projective character table for $A(7)$ corresponding to a cocycle of order six is given by the following table

$$
1 \quad(2)(4) \quad(5) \quad(7) \quad(7)^{-1}
$$

\begin{tabular}{|r|r|r|r|r|}
\hline 6 & $\sqrt{ } 2$ & 1 & -1 & -1 \\
6 & $-\sqrt{ } 2$ & 1 & -1 & -1 \\
24 & 0 & -1 & $\lambda_{1}$ & $\lambda_{2}$ \\
24 & 0 & -1 & $\lambda_{2}$ & $\lambda_{1}$ \\
36 & 0 & 1 & 1 & 1 \\
\hline
\end{tabular}

It therefore follows that the decomposition matrix is

$$
\left[\begin{array}{llll}
1 & 0 & 0 & 0 \\
1 & 0 & 0 & 0 \\
0 & 0 & 1 & 0 \\
0 & 0 & 0 & 1 \\
1 & 2 & 0 & 0
\end{array}\right]
$$

and so the Cartan matrix is

$$
\left[\begin{array}{llll}
3 & 2 & 0 & 0 \\
2 & 4 & 0 & 0 \\
0 & 0 & 1 & 0 \\
0 & 0 & 0 & 1
\end{array}\right]
$$

\section{REFERENCES}

1. K. Asano, M. Osima and M. Takahasi, Über die Darstellung von Gruppen durch Kollineationen im Körpern der Characteristik p, Proc. Phys.-Math. Soc. Japan 19 (1937), 199-209.

2. R. Brauer and C. Nesbitt, On the modular characters of groups, Ann. of Math. 42 (1941), 556-590.

3. L. Dornhoff, Group representation theory (Dekker, 1972). 
4. J. F. Humphreys, Projective modular representations of finite groups, J. London Math. Soc. (2) 16 (1977), 51-66.

5. A. Kerber, Representations of permutation groups I (Springer-Verlag, Berlin, 1971).

6. I. Schur, Über die Darstellung der symmetrischen und der alternierenden Gruppe durch gebrochene lineare Substitionen, J. Reine Angew. Math. 139 (1911), 155-250.

Department of Pure Mathematics

UNIVERSTTY OF LIVERPOOL 